\title{
Reflets
}

Revue ontaroise d'intervention sociale et communautaire

\section{Réflexions des thérapeutes familiaux francophones de la région d'Ottawa-Carleton sur la famille franco-ontarienne}

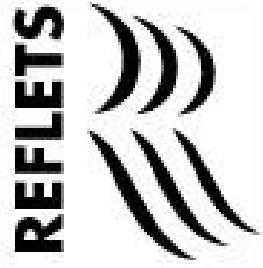

\section{Mirelle Gélinas}

Volume 1, numéro 1, printemps 1995

Des pratiques à notre image : défis et ressources

URI : https://id.erudit.org/iderudit/026055ar

DOI : https://doi.org/10.7202/026055ar

Aller au sommaire du numéro

Éditeur(s)

Reflets : Revue ontaroise d'intervention sociale et communautaire

ISSN

1203-4576 (imprimé)

1712-8498 (numérique)

Découvrir la revue

Citer cet article

Gélinas, M. (1995). Réflexions des thérapeutes familiaux francophones de la région d'Ottawa-Carleton sur la famille franco-ontarienne. Reflets, 1(1), 70-91. https://doi.org/10.7202/026055ar
Résumé de l'article

Cette étude part du point de vue que la thérapie familiale est une activité politique, basée sur des intérêts, une idéologie et des relations de pouvoir. L'auteure a analysé le discours de seize thérapeutes familiaux dans la région d'Ottawa-Carleton. Elle dégage différents enjeux socio-politiques qui sous-tendent la pratique de la thérapie familiale en Ontario français. Les discours analysés dénotent le caractère hétérogène de la communauté francophone, lequel reflète les diversités de la structure sociale ontarienne.

Elle suggère des pistes possibles pour un renouvellement des pratiques de thérapie familiale en contexte minoritaire.
Tous droits réservés (C Reflets : Revue ontaroise d'intervention sociale et communautaire, 1995
Ce document est protégé par la loi sur le droit d'auteur. L'utilisation des services d'Érudit (y compris la reproduction) est assujettie à sa politique d'utilisation que vous pouvez consulter en ligne.

https://apropos.erudit.org/fr/usagers/politique-dutilisation/ 


\section{Réflexions des thérapeutes familiaux francophones de la région d'Ottawa-Carleton sur la famille franco-ontarienne}

Cette étude part du point de vue que la thérapie familiale est une activité politique, basée sur des intérêts, une idéologie et des relations de pouvoir.

L'auteure a analysé le discours de seize thérapeutes familiaux dans la région d'OttawaCarleton. Elle dégage différents enjeux socio-politiques qui sous-tendent la pratique de la thérapie familiale en Ontario français. Les discours analysés dénotent le caractère hétérogène de la communauté francophone, lequel reflète les diversités de la structure sociale ontarienne.

Elle suggère des pistes possibles pour un renouvellement des pratiques de thérapie familiale en contexte minoritaire.

\section{Mirelle Gélinas \\ Travailleuse sociale \\ Centre d'étude de l'enfant, Université d'Ottawa}

Cet article est tiré d'une étude effectuée au printemps 1994, qui visait à amorcer une réflexion sur la pratique de la thérapie familiale auprès des familles franco-ontariennes de la région d'OttawaCarleton ${ }^{1}$. Pour mieux situer la lectrice et le lecteur face au contexte plus global de cette étude, je tracerai d'abord les grandes lignes de son cadre théorique, construit sur une analyse des principaux courants en thérapie familiale. La méthodologie utilisée pour effectuer la cueillette et l'analyse des données sera brièvement décrite et quelques thèmes axés sur ces données seront ensuite 
développés. Finalement, je présenterai certaines des questions et des pistes de réflexion qui ont émergé de ce processus de recherche.

\section{Cadre théorique}

L'étude à la base de cet article développait le point de vue que la pratique de la thérapie familiale est basée sur une théorie et des concepts traversés par les valeurs sociales dominantes et qu'elle risquait ainsi de ne pas tenir compte des valeurs et de la manière d'être des groupes minoritaires et marginalisés ${ }^{2}$. Afin de mieux saisir l'idéologie qui soustend la théorie et la pratique de la thérapie familiale, j'ai choisi d'analyser son discours, en articulant ses énoncés sur des événements socio-économiques, afin de mettre en lumière les enjeux et les rapports de force qui sous-tendent son apparition, son développement et ses transformations en tant que mode d'intervention. Quatre grands courants idéologiques ont ainsi été identifiés ${ }^{3}$, soit : la construction du savoir dans les années cinquante et soixante, le développement de techniques efficaces dans les années soixante-dix, le mouvement constructiviste des années quatre-vingt et le mouvement post-moderne des années quatre-vingt-dix.

Ces quatre courants de pensée, quoiqu'originant à différentes époques et sous-tendant des modalités de pratiques différentes, coexistent néanmoins dans la pratique actuelle de la thérapie familiale. Les différences dans la pratique s'articulent autour de plusieurs thèmes, notamment l'idéologie de la famille, la perception du rôle des thérapeutes, la définition des problèmes et des solutions ainsi que la gestion du processus de thérapie et de l'information.

De plus, chacun de ces courants de pensée adopte un point de vue différent sur la question de l'intervention auprès de groupes culturels minoritaires. Le premier courant, tout en reconnaissant l'importance des différences culturelles, préconise que les thérapeutes facilitent l'adaptation des minorités culturelles à un modèle familial dit fonctionnel, construit à partir des valeurs et 
Reflets

"Ce courant de pensée préconise la mise en relief $d u$ savoir local des personnes et la prise en considération du contexte socioculturel dans le processus de thérapie." de la manière d'être d'une population nord-américaine anglophone et de classe moyenne ${ }^{4}$. Le deuxième courant met l'emphase sur l'expertise des thérapeutes et préconise une position de neutralité. L'aspect culturel est peu développé puisque la thérapie est centrée sur l'élimination des symptômes, en ayant recours à la plupart des concepts développés dans le premier courant et en classant les familles rencontrées selon un modèle familial fonctionnel pré-établi. Le troisième courant, bien qu' il ne se réfere pas à un modèle familial fixe, demeure très influencé par les concepts traditionnels de la thérapie familiale. Il préconise la neutralité des thérapeutes et accorde peu d'attention au contexte social et culturel. Le quatrième mouvement est le seul à proposer la remise en question du principe de neutralité en thérapie et à faire une critique des concepts de base de la thérapie familiale. Ce courant de pensée préconise la mise en relief du savoir local des personnes et la prise en considération du contexte socio-culturel dans le processus de thérapie. En se basant sur cette analyse, la recherche avait donc pour objectif de découvrir comment les thérapeutes familiaux francophones de la région se situent par rapport à ces quatre grands courants idéologiques, quelles sont leurs perceptions de la réalité franco-ontarienne et comment ils et elles en tiennent compte dans leur pratique thérapeutique.

Le présent article s'attarde plus particulièrement aux perceptions des thérapeutes au sujet de la famille franco-ontarienne. Ces perceptions s'articulent autour de trois thèmes importants s'étant dégagés de l'analyse des mouvements de thérapie familiale et sous-tendant les pratiques actuelles, soit l'idéologie de la famille, la définition des problèmes et la gestion de l'information.

\section{Définition de la thérapie familiale}

La définition de la thérapie familiale sur laquelle s'appuie ma recherche s'inspire de Hartman \& Laird (1987) selon qui cette pratique consiste à rencontrer les personnes éprouvant des difficultés, non pas individuellement, mais avec un ou des membres de leur famille proche ou éloignée, afin de faciliter la résolution de situations définies comme étant problématiques par les personnes concernées. 
Le terme «thérapeute familial» désigne dans cette étude, les intervenantes et intervenants qui pratiquent la thérapie familiale ${ }^{5}$.

\section{Définition dela communauté franco-ontarienne}

"Il existe dans les écrits plusieurs définitions de la communauté francoontarienne.»
Il existe dans les écrits plusieurs définitions de la communauté franco-ontarienne. Certaines personnes utilisent le terme pour désigner tous les francophones de l'Ontario indépendamment de l'origine culturelle (Lapointe, 1988). D'autres proposent que «...la communauté franco-ontarienne s'élargisse pour inclure tous ceux qui s'y identifient, y compris ceux qui ne parlent pas ou plus le français.» (Carrière, 1993:335). La majorité des auteures et auteurs, par contre, limitent l'utilisation du terme au groupe de personnes de souche canadienne-française ayant vécu depuis plusieurs générations en Ontario (Jaenen, 1993).

Les participantes et participants à ma recherche optent en très grande majorité pour la dernière définition (81 \%); les arguments fournis pour privilégier la dimension historique du terme «FrancoOntarien» sont les suivants:

- Le terme «Franco-Ontarien» procure aux Canadiens français de l'Ontario un espace pour s'identifier à un groupe de personnes ayant la même histoire, le même vécu, la même culture.

- Le terme «Franco-Ontarien», lorsqu'appliqué à d'autres groupes culturels, risque de faire disparaitre leur appartenance à un héritage culturel et à un groupe linguistique distinct.

- Le terme «francophonie de l'Ontario» par contre semble avoir plus de potentiel pour réunir des groupes ayant une langue en commun mais une histoire, une culture et un vécu différent.

J'ai donc opté pour une définition culturelle et historique plutôt que purement linguistique de la communauté franco-ontarienne. Le terme "Canadien français» quant à lui est utilisé pour désigner l'ensemble des personnes d'origine canadienne-française vivant dans diverses provinces du Canada ou aux États-Unis. Enfin, le 
Reflets

terme «Francophones de l'Ontario» désigne tous les groupes dont la langue d'usage est le français.

\section{Recension desécrits}

Parmi les écrits recensés, deux études m'ont semblé importantes. La première, par Régis Langelier, fut effectuée en 1982 auprès d'intervenants travaillant avec des familles franco-américaines (Langelier, 1982). Cette étude visait à faciliter l'intervention auprès des familles franco-américaines en faisant ressortir des comportements et réactions typiques, et en suggérant des modalités d'intervention à l'intérieur du cadre théorique traditionnel de la thérapie familiale. La deuxième étude, par la Fédération des femmes canadiennes françaises (FFCF, 1983), rapporte que les intervenantes et intervenants rencontrés par la FFCF décrivent les femmes franco-ontariennes comme étant plus passives et acceptantes face aux services qu'elle consultent, et plus soumises aux pressions familiales et religieuses, que leurs consoeurs anglophones (FFCF, 1983). Cette étude, contrairement à la recherche de Langelier, souligne le fait que l'intervention auprès des femmes et des familles franco-ontariennes peut encourager de façon subtile cette passivité et cette soumission, ou au contraire, stimuler l'affirmation de soi et l'affranchissement des femmes face aux conventions sociales et aux traditions religieuses.

\section{Méthodologie}

Puisqu'il s'agissait d'une première étude sur le sujet, la cueillette des données concernant la pratique de la thérapie familiale auprès des familles franco-ontariennes a été effectuée par l'intermédiaire d'une méthode de recherche qualitative exploratoire (Tripodi, Fellin \& Meyer, 1985). Les données ont été recueillies lors d'entrevues semi-structurées (Mayer \& Ouellet 1991), avec 16 thérapeutes familiaux francophones de la région d'OttawaCarleton, à partir d'un échantillon par quota (Mayer \& Ouellet 1991), basé sur les différents types d'organismes offrant des services de thérapie familiale en français dans la région. 
Les entrevues ont été structurées autour de trois axes principaux: la description par les participantes et participants de leur pratique de thérapie familiale à partir des paramètres identifiés dans le cadre théorique; l'exploration de la perception qu'ont les thérapeutes participants des familles franco-ontariennes rencontrées dans leur pratique et l'exploration de la manière dont les thérapeutes tiennent compte de la réalité franco-ontarienne. Parmi les nombreux thèmes ayant émergé de l'analyse des données, ceux qui sont développés ici occupaient une place centrale et se situaient à la rencontre de points de vue divergents. L'analyse tente, à travers ces thèmes, de faire ressortir les relations de pouvoir et les enjeux inscrits dans le discours des thérapeutes.

\section{Sujets et objets du discours}

Seize thérapeutes familiaux, femmes $(60 \%)$ et hommes (40 \%), de formations professionnelles variées, ayant en moyenne 13 années d'expérience, ont participé à l'étude. Les thérapeutes rencontrés étaient majoritairement de race blanche et de souche canadienne-française $(93 \%)$. Seulement un petit nombre de participantes et participants (18\%), s'identifiaient clairement à la communauté franco-ontarienne. La situation géographique de la région d'Ottawa-Carleton fait en sorte qu'un grand nombre de thérapeutes familiaux francophones pratiquant dans cette région habitent l'Outaouais québécois. Certains thérapeutes vivent en Ontario depuis plusieurs années, mais ne se disent pas Franco-Ontariens. Les personnes rencontrées qui s'identifient spontanément à cette communauté sont nées et ont grandi en Ontario.

L'objet de ce discours sur la pratique de la thérapie familiale est la famille franco-ontarienne de la région qui consulte les services psychosociaux. Les familles francophones d'autres souches culturelles sont encore peu représentées parmi la clientèle de thérapie familiale. En effet $75 \%$ des participants/es disent ne rencontrer que rarement des personnes d'autres races et cultures. Certains thérapeutes proposent que cette absence peut être due aux facteurs suivants: les personnes d'autres races et cultures ont 
Reflets

possiblement une perspective différente des problèmes rencontrés, donc abordent ces difficultés avec des moyens qui conviennent davantage à leur vision du monde; ces personnes se méfient peutêtre des interventions publiques dans leurs affaires personnelles, elles ont peur d'être incomprises et jugées par les étrangers qui offrent les services; elles sont moins référées aux services de thérapie et plus référées aux services de justice et de contrôle social. (ex: les adolescents d'origine afro-canadienne sont plus souvent perçus comme étant délinquants que comme ayant des problèmes émotifs.)

\section{Les thèmes et leur analyse}

\section{La réalité franco-ontarienne: le mythe de la grande famille unie}

Le discours sur la préservation de la langue qui brandit le spectre de l'assimilation, tend à présenter la communauté francoontarienne comme une grande famille dont les intérêts sont communs. Une communauté minoritaire constamment menacée

"Le discours sur la préservation de la langue... tend à présenter la communauté franco-ontarienne comme une grande famille dont les intérêts sont communs.» d'extinction éprouve le besoin de se protéger des risques de divisions internes qui pourraient l'affaiblir et de conserver ses énergies pour lutter contre l'ennemi véritable. Les conflits d'intérêts à l'intérieur de la communauté risquent ainsi d'être occultés, et les groupes défavorisés par le système social actuel risquent d'être sacrifiés à la grande cause. La bannière de cette grande cause est souvent portée par une élite dont l'expérience est différente de celle des Franco-Ontariennes et Franco-Ontariens ordinaires ou défavorisés (Roberts, 1979; Martel, 1993).

Les thérapeutes familiaux de la région participant à cette étude ont pour la plupart éprouvé de la difficulté à identifier des caractéristiques propres à l'ensemble des familles francoontariennes qu'ils rencontrent dans leur pratique. Les thérapeutes ont en très grande majorité fait des distinctions importantes entre les groupes qui occupent une position plus centrale et privilégiée, et ceux qui sont plutôt en périphérie et défavorisés à l'intérieur même de la communauté franco-ontarienne ou par 
«La famille francoontarienne ne peut donc pas être traitée en bloc en utilisant la culture et la langue comme uniques points de référence.» rapport à la société dominante nord-américaine anglophone. La famille franco-ontarienne ne peut donc pas être traitée en bloc en utilisant la culture et la langue comme uniques points de référence.

\section{Les parents pauvres}

"...Je fais une distinction entre deux groupes de gens; les gens qui sont de classe moyenne ou haute: ceux-là ont beaucoup évolué dans le milieu anglophone, ont tendance à avoir une plus grande éducation, plus de facilité à se procurer des services et à se sentir à la hauteur du milieu social. Tandis que les classes défavorisées moins éduquées, c'est là où je vois une grosse différence.»

Le statut économique est un des facteurs qui influence grandement l'accès à la parole et au pouvoir parmi les membres de la communauté franco-ontarienne. Plusieurs thérapeutes familiaux de la région perçoivent par exemple que les Franco-Ontariennes et Franco-Ontariens à l'aise financièrement ont plus facilement accès à une variété de services; que les approches de thérapie familiale correspondent davantage à leur manière d'être; qu'ils sont moins intimidés par la société dominante anglophone; que le lien entre les thérapeutes et ces personnes est plus facile, puisque les conditions de vie et la vision du monde apparaissent comme étant plus semblables.

Par contre, les personnes financièrement démunies qui consultent les services risquent de se sentir dévalorisées et intimidées dans la relation thérapeutique, qui ne peut faire autrement qu'être colorée par les différences de pouvoir, de mentalité et de points de vue entre «l'élite» professionnelle qui aide et «la famille pauvre» qui demande de l'aide. Peu de thérapeutes ont abordé la question de ces différences qui font partie intégrante de la relation thérapeutique, quelle que soit l'attitude personnelle des thérapeutes. Quelques-uns ont cependant soulevé la difficulté pour les personnes financièrement démunies, de s'engager et de cheminer dans un processus de thérapie familiale. 
Reflets

«Les réseaux naturels

qui s'étaient formés

dans certains quartiers

sont souvent détruits

par le processus de

planification urbaine.»
"...Ce qu'on offre c'est du beau gros luxe de classe moyenne et on essaie de faire gober ça aux démunis et ça marche pas...»

"...Avec les familles défavorisées ma façon de faire sera la même excepté que je vais m'adapter à leur niveau... C'est des familles qu'on va suivre plus à long terme, parce qu'elles ont besoin d'être suivies...”

\section{Les parents de la campagne}

«...Dans les petits villages, c'est l'Église, les clubs. C'est une autre mentalité. Ces familles-là vont se tourner vers les regroupements communautaires avant de venir consulter ici... Il y a toujours une connotation religieuse..."

Plusieurs thérapeutes ont souligné que les gens de la campagne restent souvent plus attachés aux traditions, sont plus près de la famille élargie, des groupes communautaires, et des réseaux paroissiaux. Ils ont souvent tendance à se référer à ces ressources lorsqu'ils ou elles éprouvent des difficultés dans leur vie familiale, plutôt que de consulter les professionnelles et professionnels du secteur social. Ils sont assujettis à ces différents groupes qui exercent des pressions importantes pour les inciter à se soumettre à certaines conventions sociales et communautaires et à certaines normes de comportement (FFCF, 1983; Welch, 1993).

Par contre, particulièrement dans la région d'Ottawa-Carleton, les familles urbanisées sont souvent séparées de leur famille élargie, et sont peu liées avec la paroisse et avec les groupes communautaires. Plusieurs thérapeutes ont mentionné l'absence de vie familiale et communautaire et l'isolement qui en résulte comme étant un problème important pour les familles franco-ontariennes qui viennent les consulter. Les réseaux naturels qui s'étaient formés dans certains quartiers sont souvent détruits par le processus de planification urbaine (Welch, 1994). 
"En ville, le tissu communautaire est très effiloché. Dans les villages, c'est encore important le curé du village, il y a une communauté...”

"C'est peut-être que dans notre société on a pas beaucoup d'amis, pas tellement de gens à qui parler. Les femmes sont isolées, il n'y a plus de famille... la thérapie peut dans certains cas devenir un moyen d'avoir quelqu'un sur qui compter, qui nous soutient...”

Cet isolement que vivent un grand nombre de familles francoontariennes pousse les gens à utiliser les services psycho-sociaux pour obtenir de l'aide, des conseils, de l'appui. Ce mouvement vers les services publics entraîne un foisonnement des services francophones dans la région. Les professionnelles et professionnels du secteur social et des agences qui doivent continuellement justifier leur existence bénéficient possiblement de ces nouveaux services. Est-ce que la communauté franco-ontarienne en bénéficie? La réponse à cette question est plus douteuse.

\section{Pour le meilleur et pour le pire...}

«...Les fermmes sont pauvres... les fermmes monoparentales... beaucoup sont sur le bien-être social. Si elles vont à l'école ou si elles travaillent un peu, on les coupe..."

À travers l'histoire, les femmes canadiennes-françaises ont porté le poids de la reproduction et de la sauvegarde de la race, de la préservation de l'unité familiale et du bien-être physique et émotionnel des enfants et des hommes. Les activités sociales féminines ont longtemps été centrées autour de la famille, de la religion, de la langue et de la culture, de sorte que leur évolution personnelle a souvent été sacrifiée ou mise en attente (Cardinal 1993). Dans la grande famille franco-ontarienne, les femmes continuent d'être moins valorisées et moins payées que leurs confrères masculins. Une étude publiée par Marc Lavoie et Maurice St-Germain en 1990 situe le niveau de revenu des femmes francophones de 45 à 54 ans ayant une éducation au niveau universitaire à 34557 \$ pour l'année 1985, tandis que celui des hommes francophones 
Reflets

du même groupe se situe à 56329 \$. (Lavoie et St-Germain, 1990:144)

"...les femmes sont

plus présentes dans

les services psycho-

sociaux que les

hommes.»
Plusieurs des thérapeutes rencontrés soulignent que les femmes sont plus présentes dans les services psycho-sociaux que les hommes. Ceci semble indiquer entre autres, qu'elles se définissent plus facilement comme ayant des difficultés, qu'elles se sentent plus impliquées dans le bien-être émotionnel de leurs proches et qu'elles se perçoivent comme étant responsables des changements à apporter à leur situation familiale.

\section{L'idéologie de la famille: la sainte famille remise en question.}

$$
\begin{aligned}
& \text { "... Malheureusement,...dans ma pratique... il y a } \\
& \text { beaucoup de familles éclatées..." } \\
& \text { "...Il n'y a plus de racines, de stabilité dans les fa- } \\
& \text { milles... ce n'est pas rare qu'un enfant va dire 'j'ai } \\
& \text { deux pères, deux mères'..." }
\end{aligned}
$$

C'est sur l'institution familiale régie par l'Église qu'a longtemps reposé la survivance de la langue et de la culture canadiennefrançaise. La famille traditionnelle canadienne-française est souvent présentée dans la littérature comme une sorte de lieu sacré qui se protège des influences extérieurs en se refermant, et qui ne supporte pas les conflits et différences internes qui pourraient menacer son intégrité (Langelier, 1982). Les femmes sont souvent celles qui héritent ce rôle de gardienne des familles; celles qui ne remplissent pas ce rôle sont décrites comme des «mères martyres manipulatrices» (Langelier, 1982) et des grands-mères «contrôlantes» et «sur-impliquées».

La plupart des thérapeutes familiaux interrogés soulignent l'importance des liens familiaux dans la culture franco-ontarienne et le fait que les familles rencontrées dans leur pratique ont souvent moins accès au support de la famille élargie. Plusieurs thérapeutes notent que la mère franco-ontarienne occupe souvent une position centrale dans la famille. Cette centralité de la mère est perçue par certains thérapeutes comme l'indice d'un certain pouvoir 
"Les thérapeutes réfêrent souvent à la famille biparentale traditionnelle comme étant plus stable, mieux adaptée aux besoins des enfants, moins problématique que la famille monoparentale ou reconstituée.» qu'elle exerce dans l'unité familiale, mais plus souvent comme étant un signe du poids des responsabilités que la mère francoontarienne continue de porter.

Par contre, les thérapeutes remarquent que le rôle traditionnel $\mathrm{du}$ père "protecteur, pourvoyeur et chef du foyer» est remis en question dans les familles reconstituées et les familles de classe moyenne. De plus, les femmes sont souvent chefs de famille et le père est parfois absent. Les relations familiales sont plus fluides et ouvertes et incluent souvent un grand nombre de personnes qui auraient autrefois été considérées des «étrangers».

Le modèle familial de type traditionnel tend cependant à être reproduit et encouragé. La famille biparentale hétérosexuelle, par exemple, est un idéal auquel la plupart des thérapeutes et des personnes qui consultent semblent continuer d'adhérer. Les thérapeutes réferent souvent à la famille biparentale traditionnelle comme étant plus stable, mieux adaptée aux besoins des enfants, moins problématique que la famille monoparentale ou reconstituée. Certains thérapeutes soulignent également que les Franco-Ontariens et Franco-Ontariennes continuent d'aspirer au modèle familial traditionnel parce que c'est le modèle le plus viable financièrement et socialement dans la société actuelle de consommation, où deux salaires sont nécessaires pour survivre.

«... La famille reconstituée c'est la nouvelle famille... les femmes ne restent pas seules... elles savent que si elles prennent un nouveau conjoint elles peuvent s'acheter une maison..."

"... Ça prend deux salaires pour arriver de nos jours... Les familles monoparentales sont épuisées par le travail, les enfants et les pressions économiques...»

Les thérapeutes notent que les familles franco-ontariennes subissent de plus en plus de pressions économiques et qu'un très grand nombre d'entre elles éprouvent de la difficulté à se procurer l'essentiel. Les familles qui sont à l'aise financièrement se trouvent sous l'influence d'une société où l'acquisition de biens matériels et le succès au travail constituent la mesure de la valeur des 
Reflets

personnes. Ces pressions économiques et sociales, combinées avec l'absence de support familial et communautaire font partie des difficultés que rencontrent les familles qui consultent les services psycho-sociaux.

\section{Un peuple soumis?}

La communauté franco-ontarienne a longtemps accepté l'autorité et l'ingérence de l'Église catholique dans la famille, mais elle a appris à se méfier de l'ingérence de l'État anglophone. Et les professionnelles et professionnels du secteur social sont des agents de l'État. Le discours des thérapeutes familiaux francophones de la région révèle que les familles franco-ontariennes ne vont pas souvent en thérapie familiale de leur propre initiative. La plupart des thérapeutes rencontrés soulignent que les familles sont en grande partie référées, souvent par le système scolaire qui a identifié un enfant ou adolescent problématique.

"...Si j'ai vu dix familles en trois ans c'est beau... les demandes ne sont pas là. On a beaucoup de demandes en individuel et environ $80 \%$ sont des femmes...»

"...La majorité accepte de venir en famille... ils ne le demandent pas, je dois expliquer...»

...elles sont souvent référées par l'école...

Les participantes et participants à l'étude sont généralement d'avis qu'une fois engagés dans un processus de thérapie, les FrancoOntariennes et Franco-Ontariens sont peu enclins à questionner ouvertement les professionnelles et professionnels. La compilation de dossiers et la formulation d'évaluations et d'impressions cliniques sont des activités bureaucratiques largement acceptées aussi bien par les thérapeutes que par les personnes qui demandent de l'aide. La plupart ne remettent pas une copie des rapports d'évaluation effectués aux personnes concernées. Beaucoup de thérapeutes n'en voient pas l'utilité tandis que d'autres ont tendance à décourager les personnes qui en font la demande. 
"....s'ils demandent de voir l'évaluation ou le dossier, je vais plutôt les questionner à savoir pourquoi c'est important pour eux..."

Quelques thérapeutes ont par contre exprimé leur regret face à la soumission des personnes qui consultent, envers le système.

"...Les gens ont tendance à respecter le système. Ils ne demandent pas au sujet des dossiers..."

"...Les gens ne se préoccupent pas de ça... ils sont habitués que les professionnels cachent tout...»

Il semble y avoir ici une exception: les gens travaillant dans le domaine médical. Ces derniers rapportent être de plus en plus remis en question par les personnes qui les consultent.

«...Les gens sont plus sophistiqués maintenant, même les gens démunis. Ils questionnent plus le diagnostic et le traitement... si quelqu'un me demande ce que j'ai mis dans l'évaluation je vais dire: corrigez-là et ramenez-la moi...»

Ce questionnement du savoir professionnel qu'on remarque de plus en plus dans le domaine médical, ne semble pas exister au même degré dans le domaine psychologique. Il est possible que les gens qui éprouvent des difficultés psycho-sociales se sentent plus vulnérables et font moins confiance à leur propre jugement et à leur propre autorité. Le moyen le plus répandu dans ce domaine de protester contre l'évaluation et le traitement est d'abandonner la thérapie.

\section{La définition des problèmes: l'effet rétrécissant de la thérapie}

"... Quand la mère arrive à la fin du mois et qu'il n'y a plus rien à manger, elle est en état de survie... je ne pense pas qu'elle peut se concentrer sur l'interaction avec son enfant. C'est difficile d'arriver aux vrais problèmes...» 
Reflets

"La thérapie risque

donc... de devenir un

instrument pour

faciliter l'adaptation

des personnes de

certains groupes à un

système qui les

opprime.»
La pratique de la thérapie familiale offre une analyse plutôt étroite de la conceptualisation des problèmes auxquels les gens font face. Les thérapeutes et les personnes qui consultent sont invités à définir les problèmes d'ajustement psycho-social comme étant soit à l'intérieur des personnes ou entre les membres de leur famille immédiate ou élargie. Les problèmes d'ordre psychodynamique et d'ordre familial sont perçus comme étant les vrais problèmes ou tout au moins les problèmes qui peuvent être abordés dans le contexte de la thérapie. Les problèmes structurels comme la pauvreté et la marginalisation sont présentés comme étant des facteurs importants mais qui empêchent ou contribuent à ralentir l'accès aux problèmes psychologiques et interpersonnels qui peuvent être traités en thérapie.

La thérapie familiale tend donc à ignorer les facteurs historiques $^{6}$, culturels, économiques et sociaux des problèmes. En effet, bien que ces facteurs aient été soulevés par beaucoup de participantes et de participants comme jouant un rôle important dans les problématiques rencontrées, on note qu'ils sont difficiles à inclure dans le cadre étroit de la thérapie familiale. La thérapie risque donc, par le fait qu' elle exclut ces dimensions, de devenir un instrument pour faciliter l'adaptation des personnes de certains groupes à un système qui les opprime. La thérapie familiale peut avoir tendance à inciter les personnes à s'isoler dans leur difficultés, soit individuellement ou en famille, plutôt que de chercher à s'allier à d'autres personnes dans leur communauté pour lutter ensemble face à un problème commun.

\section{L'invisibilité de la culture franco-ontarienne}

La plupart des thérapeutes familiaux rencontrés lors de cette étude ont éprouvé de la difficulté à définir la spécificité de la culture franco-ontarienne en dehors de la langue parlée et à expliquer comment ces aspects culturels pouvaient être intégrés dans le cadre de la thérapie familiale. Il est possible que cette difficulté soit rattachée au fait que la majorité des thérapeutes rencontrés demeure dans la province de Québec ou ne s'identifie pas à la communauté franco-ontarienne. Il est possible aussi que la population francophone d'Ottawa-Carleton ne soit pas typiquement 
«Il semble que la culture francoontarienne soit difficile à saisir pour les gens qui n'appartiennent pas à ce groupe.» franco-ontarienne puisqu'elle comprend beaucoup d'éléments provenant d'autres provinces. Quelle que soit la raison, la plupart des thérapeutes (exception faite des thérapeutes franco-ontariens) ont perçu très peu de différence entre la population francoontarienne et la population québécoise, en dehors de la langue parlée. Quelques thérapeutes ont également exprimé l'opinion qu' ils percevaient peu de différence entre la culture moderne canadienne-française et la culture nord-américaine en ce qui concerne les problèmes, les défis à relever pour les familles et les solutions à apporter aux problèmes.

Il semble que la culture franco-ontarienne soit difficile à saisir pour les gens qui n'appartiennent pas à ce groupe. De leur côté, les thérapeutes d'origine franco-ontarienne ont exprimé que c'est une histoire et un vécu communs qui leur donnent leur identité, plutôt qu'un ensemble de caractéristiques communes. Étant donné cette invisibilité de la culture franco-ontarienne, peu de thérapeutes ont exprimé ressentir le besoin d'adapter les concepts traditionnels de la thérapie familiale aux familles qu'ils rencontrent dans leur pratique.

Certains thérapeutes ont cependant noté que ce n'est pas tant l'approche utilisée qui est différente, mais la qualité de la relation entre les thérapeutes et les familles qui partagent la même langue et la même culture de base. C'est comme de «...comprendre la famille par le dedans...", exprime une thérapeute franco-ontarienne. «...Je suis plus naturel, j'hésite moins à utiliser le langage du peuple... je me retrouve dans la culture... je peux saisir les distinctions, les accents de différentes régions...", rapporte une autre intervenante.

\section{Questions et réflexions découlant du processus de recherche}

Cette analyse m'invite à poser certaines questions au sujet du contrôle de ses institutions psycho-sociales par la communauté franco-ontarienne ainsi qu' à réfléchir aux implications possibles 
Reflets

de cette étude pour la pratique de la thérapie familiale auprès des familles franco-ontariennes.

\section{Contrôle des institutions ou contrôle du savoir}

Dans les services psycho-sociaux francophones de la région d'Ottawa-Carleton, particulièrement dans le domaine de la thérapie familiale, il semble y avoir une pénurie de thérapeutes franco-ontariens. Il est également rare de rencontrer des thérapeutes francophones d'autres groupes ethniques. Est-ce que les services francophones de la région d'Ottawa-Carleton tendent à exclure certains groupes et à en avantager d'autres?

Les modèles théoriques et pratiques de thérapie familiale ont été développés en grande partie par et pour une population nordaméricaine anglophone et de classe moyenne. Si la communauté franco-ontarienne doit reprendre le contrôle de ses institutions psycho-sociales, n'est-il pas important qu'elle entreprenne de réexaminer le savoir véhiculé par ses pratiques de thérapie, afin de s'assurer qu'il soit sympathique à la manière d'être francoontarienne?

Beaucoup de services psycho-sociaux francophones sont déployés pour une population démunie. Les thérapeutes prétendent que cette population est choyée de pouvoir rencontrer des professionnelles et professionnels qui parlent sa langue et partagent sa culture. Il est pourtant essentiel de ne pas oublier, comme l'ont mentionné quelques participants, que la distance sociale entre les thérapeutes et ces personnes n'est pas pour autant abolie, et que cette population continue à exercer très peu de contrôle sur les institutions francophones qui la desservent. N'existe-t-il pas un risque que l'élite professionnelle francophone utilise à son insu, ses "parents pauvres» franco-ontariens pour se constituer un territoire d'opération et exercer son pouvoir/savoir?

\section{Implications pour la pratique}

J'aimerais avancer le point de vue qu'en tant que thérapeutes familiaux oeuvrant auprès d'une population minoritaire, et 
"...nous risquons de participer sans nous en rendre compte à

l'oppression de cette population.»
"...La promotion des réseaux naturels

d'entraide... pourrait contribuer à redonner aux Franco-Ontariennes et Franco-Ontariens un sens d'appartenance à une communauté...» souvent même socio-économiquement défavorisée, nous risquons de participer sans nous en rendre compte à l'oppression de cette population. En effet, le cadre théorique et les concepts traditionnels de la thérapie familiale ont tendance à favoriser l'adaptation des personnes à la société dominante. Par contre, à l'instar de plusieurs thérapeutes du quatrième mouvement en thérapie familiale, j'aimerais également suggérer que si les thérapeutes familiaux peuvent participer à l'adaptation des personnes, ils peuvent aussi participer à leur conscientisation et à la transformation du système social (White, 1994; Tamasese \& Waldergrave, 1993; Goldner, 1985).

Une pratique thérapeutique peut être conscientisante si elle tente de garder les grands problèmes sociaux qui affectent les personnes au centre, et non en marge, de la conversation thérapeutique. Une telle pratique pourrait chercher à faire apparaitre le filet invisible des idées, croyances et présuppositions véhiculées dans l'environnement social, et qui influencent la perception des personnes et des thérapeutes au sujet de la situation présentée en thérapie.

Une conscience accrue de la part des thérapeutes des relations de pouvoir et des enjeux sociaux qui sous-tendent la relation thérapeutique pourrait les inviter à poser des gestes concrets pour rendre la relation plus égale, moins opprimante. Par exemple, une collaboration avec les personnes dans le cas où des évaluations doivent être écrites à leur sujet; la remise automatique aux personnes de tout document formel ou informel produit à leur sujet; un certain degré de révélation de soi de la part des thérapeutes.

La promotion des réseaux naturels d'entraide plutôt que la multiplication des services offerts par l'État, pourrait contribuer à redonner aux Franco-Ontariennes et Franco-Ontariens un sens d'appartenance à une communauté plutôt qu'à les inviter à s'isoler davantage.

Bref, j'aimerais proposer que, en tant que Franco-Ontariennes et Franco-Ontariens avec nos consoeurs et confrères francophones d'autres provinces et d'autres races et cultures, nous luttions non seulement "pour les services en français» mais que nous refusions également de coopérer, par le biais de nos pratiques, avec un 
Reflets

système social qui place certains groupes de personnes au centre tandis que d'autres sont relégués dans les marges.

\section{Conclusion}

Ces réflexions sont issues d'une étude sur la thérapie familiale en relation avec la communauté franco-ontarienne de la région d'Ottawa-Carleton. L'étude à la base de cet l'article développait le point de vue que la théorie et les concepts traditionnels de la thérapie familiale sont traversés par les valeurs sociales dominantes. La thérapie familiale risque donc de participer au processus de reproduction sociale et à l'oppression des groupes minoritaires et marginalisés dans le sens qu'elle ne tient pas nécessairement compte de leurs valeurs, leur manière d'être, leur histoire et leurs intérêts en tant que groupe. À travers l'étude des écrits en thérapie familiale, quatre grands courants idéologiques faisant apparaitre des pratiques différentes étaient identifiés. L'étude visait à situer les thérapeutes familiaux de la région par rapport à ces grands courants de pratique et à amorcer une réflexion sur la famille franco-ontarienne telle que perçue par les thérapeutes.

Le présent article portait surtout sur la perception des thérapeutes familiaux francophones de la région au sujet de la famille franco-ontarienne. Ces perceptions, recueillies par le biais d'entrevues, étaient articulées autour de quelques thèmes qui sous-tendent la pratique de la thérapie familiale.

L'analyse des données recueillies a révélé que la spécificité de la culture franco-ontarienne est difficile à saisir pour des francophones n'ayant pas vécu en milieu minoritaire; que la communauté franco-ontarienne est très hétérogène et que les thérapeutes familiaux doivent éviter de la traiter en bloc; que cette communauté tend à reproduire la structure sociale de la société dominante; que les familles franco-ontariennes qui consultent les services de thérapie familiale sont particulièrement affectées par les changements dans la structure de la famille, l'isolement, la 
pauvreté et l'épuisement relié aux pressions sociales, parentales et économiques; que le contexte de la thérapie familiale s'ouvre difficilement aux aspects culturels, historiques et socio-économiques des problèmes présentés par les familles. L'article présentait également quelques questions et réflexions personnelles découlant de la démarche de recherche, et des échanges effectués avec les participants et participantes.

Il est important finalement de souligner que l'étude à la base de cet article reflète les caractéristiques particulières de la région d'Ottawa-Carleton, et qu' une étude semblable effectuée dans une autre région de l'Ontario ferait possiblement apparaitre un discours différent. L'étude reflète également la perception des thérapeutes familiaux seulement. Une étude auprès des personnes qui reçoivent les services de thérapie familiale pourrait être très intéressante, puisqu'elle permettrait aux personnes de différents milieux socio-économiques de faire valoir leur point de vue et de partager leur expérience.

\section{Notes}

1. Gélinas, M., «Analyse du discours des thérapeutes familiaux francophones de la région d'Ottawa-Carleton", projet de mémoire présenté à l'École de service social de l'Université d'Ottawa, dirigé par Roland Lecomte, août 1994.

2. Ce point de vue est développé dans le cadre analytique de l'étude à travers l'analyse des écrits en thérapie familiale, de son apparition dans les années cinquante jusqu'à nos jours. Il s'appuie également sur des critiques effectuées par divers auteurs et auteures, par exemple, Luepnitz 1988, Hare-Mustin 1978, Almeida 1993 et McLean 1990.

3. J'ai identifié ces quatre courants idéologiques en me basant sur les quatre vagues de la thérapie familiale décrites par Slovik \& Griffith (1992), sur la description des différents discours de la thérapie familiale de Harré Hindmarsh (1993), sur mon expérience personnelle en tant que praticienne dans le domaine et sur la recension des écrits. 
Reflets

4. Ackerman (1958) et Epstein (1981) réferrent eux-mêmes au fait que les concepts importants de la thérapie familiale sont basés sur le fonctionnement de familles nord-américaines anglophones et de classe moyenne.

5. Certains participants et participantes font une distinction importante entre «thérapie» et «counselling» et se perçoivent soit comme conseillères, conseillers ou thérapeutes. Pour les besoins de cette étude, je ne fais pas cette disctinction. Le terme «thérapeute» est donc utilisé pour désigner tous les intervenants et intervenantes qui ont participé à l'étude.

6. Dans le sens de l'histoire des peuples.

\section{Bibliographie}

ACKERMAN, Nathan W. (1958). The Psychodynamics of Family Life. Diagnosis and Treatment of Family Relationships, Basics Books, N.Y.

ALMEIDA, V. Rhea (1993). «Unexamined Assumptions and Service Delivery Systems: Feminist Theory and Racial Exclusions», Journal of Feminist Family Therapy, vol. 5, no 1, pp. 3-23.

CARDINAL, Linda, ed. (1993). Une langue qui pense: la recherche en milieu minoritaire francophone au Canada, Ottawa, Les Presses de l'Université d'Ottawa.

CARDINAL, Linda (1992). «Femmes et francophonie: une relecture du rapport ethnicité-féminité», Actes du Colloque: La recherche sur les femmes francophones vivant en milieu minoritaire: un état de la recherche, juin 1992.

CARRIÈRE, Fernan (1993). «Métamorphose de la communauté francoontarienne», dans Cornelius Jeanen, ed. Les Franco-Ontariens, Ottawa, Les Presses de l'Université d'Ottawa.

DESJARDINS, Micheline (pour la Fédération des femmes canadiennes francaises) (1983). Seules en grand nombre - Dossier socioéconomique sur la situation des ontaroises chefs de famille monoparentale, Ottawa, FNFCF.

DONZELOT, Jacques (1977). La police des familles, Paris, Les éditions de minuit.

DUQUETTE, Georges et Chantal CLÉROUX (1993). «Vivre en milieu minoritaire: Principes théoriques, possibilités et limites, recommandations pratiques», La Revue canadienne des langues vivantes, vol. 49, no 4, pp. 770-785.

EPSTEIN, Nathan B. et Duane S. BISHOP (1981). "Problem-Centered Systems Therapy of the Family», dans Alan Gurman et David Kniskern, ed. The Handbook of Family Therapy, New York, Brunner/Mazel, pp. 444-482.

FOUCAULT, Michel (1969). L’archéologie du savoir, Paris, Éditions Gallimard.

GOLDNER, Virginia (1985). «Feminism and Family Therapy», Family Process, vol. 24, no 1, pp. 31-47. HARE-MUSTIN, Rachel (1978). "A Feminist Approach to Family Therapy», Family Process, vol. 17, pp. 181-94. 
HARE-MUSTIN, Rachel (1987). "The Problem of Gender in Family Therapy», Family Process, vol. 26, no 1, pp. 15-27.

HARTMAN, Ann et Joan LAIRD (1987). «Family-Centered Practice», The Encyclopedia of Social Work, 18th Edition, USA, N.A.S.W.

HINDMARSH, Jennie Harré (1993). "Alternative Family Therapy Discourses: It Is Time to Reflect (Critically)", Journal of Feminist Family Therapy, Vol 5, no 2, pp. 5-27.

JAENEN, J. Cornelius, ed (1993). Les Franco-Ontariens, Ottawa, Les Presses de l'Université d'Ottawa.

LANGELIER, Régis (1982). «French-Canadian Families», dans Monica McGoldrick, John K. Pearce et Joseph Giordano, ed. Ethnicity and Family Therapy, New York, Guilford Press.

LAPOINTE, Jean (1986). «Vie culturelle et institutions autonomes: minorités culturelles et institutions», Revue du Nouvel Ontario, no 8.

LAVOIE, Marc et Maurice ST-GERMAIN (1990). «Évolution comparative des revenus des FrancoOntariens", Revue du Nouvel-Ontario, no 12, pp. 125-149.

LUEPNITZ, D.A. (1988). The Family Interpreted, USA, Harper Collins.

MARTEL, Marcel (1993). «De la certitude au doute: l'identité canadiennefrancaise de l'Ontario de 1937 à 1967», dans Linda Cardinal, ed. Une langue qui pense: la recherche en milieu minoritaire francophone au Canada, Ottawa, Les Presses de l'Université d Ottawa.

MAYER R. et F. OUELLET (1991). Méthodologie de recherche pour les intervenants sociaux, Boucherville, Gaëtan Morin Éditeur.

McLEAN, Athena (1990). «Contradictions in the Production of Clinical Knowledge:The Case of Schizophrenia», Social Science Medical, vol. 30, no 9, pp. 969-985.

SILVERSTEIN, O., M. WALTERS, B. CARTER et P. PAPP (1988). The Invisible Web - Gender Patterns in Family Relationships, New York, Guilford Press.

SLOVIK, Lois-Sims et James L.GRIFFITH (1992). "The Current Face of Family Therapy», dans Rutan J. Scott, ed. Psychotherapy for the 1990's, New-York, Guilford Press, pp. 221-244.

TAMASESE, Kiwi et Charles WALDEGRAVE (1993). "Cultural and Gender Accountability in the «Just Therapy» Approach», Journal of Feminist Family Therapy, vol. 5, no 2, pp. 29-45.

TRIPODI, T. et P. FELLIN, MEYER (1985). The Assessment of Social Research, Stasca, Peacock.

URSEL, Jane (1992). Private Lives, Public Policy: 100 Years of State Intervention in the Family, Toronto, Women's Press.

WALDEGRAVE, Charles (1990). «Just Therapy», Dulwich Centre Newsletter, no 1, pp. 5-46.

WELCH, David (1993). "Early Franco-Ontarian Schooling as a Reflection and Creator of Community Identity», Ontario History, vol. 95, no 4, pp. 321-347.

WELCH, David (1994). Communication présentée lors d'un colloque organisé par l'École de service social, Ottawa, Université d'Ottawa.

WHITE, Michael (1994). The Politics of Therapy: Putting to Rest the Illusion of Neutrality (paper presented at a conference in Vancouver, March). 